\title{
Ontology and Presentation of Cultural Heritage Management and Maintenance: A Case Study of Qiong-Lin Settlement in Kinmen
}

\author{
Y. N. Yen, W. B. Yang* \\ China University of Technology, 56 Sec. 3 ShingLong Rd., 116 Taipei, Taiwan - (alexyen, wunbin)@cute.edu.tw
}

KEY WORDS: Heritage, Management and maintenance, Ontology, CIDOC CRM, Arches

\begin{abstract}
:
The management and interpretation of cultural heritage is an important international conservation issue. The construction of longterm sustainable international exchange information will help to present and promote the interpretation of intention for the cultural heritage. The Arches system platform of this study presents the concept of the life cycle of cultural heritage, and establishes the thesauri and metadata in the management and maintenance stage of Taiwan's monuments, which can mapping to the entity of relevant ontology, covering the contents required by previous visits and related needs interviews, while the detailed description part is still filled with the entity of description; the presentation platform is no longer limited to the vertical or horizontal query of the existing national database and can integrate categories, keywords, maps and other ways to present related knowledge, and expand to the system according to a physical thing, actor, activity and other entities, so as to enhance the functionality of user search. We should make long-term planning to establish complete metadata and ontology model of the tangible and intangible life cycle, provide the development direction of the national cultural heritage database and provide the basic work of international data exchange in the future.
\end{abstract}

\section{INTRODUCTION}

One of the important keys to the dissemination and sharing of cultural heritage data is the establishment of metadata, which can be used as the data standard of cultural heritage database content and the interpretation function of data exchange in accordance with international standards. Combined with the interpretation data standard established by ontology, the interpretation data has semantic relevance, which can strengthen the presentation of the diversity of interpretation data; it also has the function of international data exchange and promotion.

The international digitized data exchange method is mainly based on the establishment of metadata standards, such as the Eurpoeana digital platform, which is the main European cultural heritage. The initial construction of the platform uses Dublin Core as the metadata structure, which has limited basic fields and contents. However, to improve the lack of data content and enhance the semantic relevance of interpreting data, European and American countries have used ontology as the main application and development trend on relevant platforms in recent years. On the contrary, the main management system of cultural heritage in Asia, such as Japan and China, is still based on a simple query of DC architecture.

Although the current National Cultural Heritage Network in Taiwan adopts Dublin Core as the standard for the interpreting data, so that the standard is too simplified and lacks more detailed and relevant data, which makes the data unable to be described in depth. This study takes the long-term development demands of cultural heritage information architecture, and cooperates with international trends to establish an interpretive database based on ontology standards, as well as an important basis for data exchange, as an important tool to promote the aforementioned preservation and education promotion strategies. The synchronization of international development trends is of great significance.

In view of the long-term development of cultural heritage information architecture, the content of cultural heritage description data should further establish an ontology based interpretation data standard. This study intends to add the current international standard CIDOC CRM ontology as the interpretation data standard based on the current cultural heritage website in Taiwan, so as to make the final database system conform to the international cultural heritage exchange standard, it is the first step to achieve the data in line with international standards.

\section{BACKGROUND}

Taiwan has been actively promoting the preservation and management of cultural heritage. Under the condition that cultural heritage need to be preserved and managed, the number of designated registration has increased year by year. By June 2021, there are 5739 cultural heritage; especially 987 designated historical settlement, which require a large number in the preservation of cultural heritage. The cumbersome and complicated preservation procedures, can easily lead to inconvenience in file management, inconsistent formats, and the dilemma of improving management efficiency.

In recent years, the implementation results have mostly focused on digital records and modeling presentations; the established metadata benchmarks are mostly used as basic descriptions for the purpose of archiving, but the overall architecture and management functions still need to be continuously deepened. However, the national cultural heritage network focuses more

\footnotetext{
* Corresponding author
} 
on the announcement of basic information, and there is no complete framework for the ontology relevance of cultural heritage, which will form a closed system and be unable to effectively integrate with international standards.

CIDOC CRM is a well-defined ontology model for the integration and preservation of cultural resources in the current ontology. The main purpose of CIDOC CRM is to implement the data exchange and integration of various cultural heritage data from different sources. In 2006, it became ISO 21127 standard, which is the most widely used model of cultural heritage integration ontology (Ronzino, 2013; Moraitou, 2019). CIDOC CRM is characterized by a wide range of applications. There are many cultural heritage ontologies that use CIDOC CRM as a standard in the world. However, the purpose, reference materials, consulting experts, and requirements of each ontology are not the same, so we need to pay attention to the differences.

Doerr (2009) mentioned that due to the difference between the field of cultural heritage and the field of information engineering, it is very inefficient to build a complete conceptual model by relying on a single aspect. Since the development of CIDOC CRM in 1995, experts from various fields have been invited many times in the process. At present, it has become the most refined model for the integration of cultural heritage ontology, although the details of the case may not be enough, but most of the concepts have been taken into consideration.

In order to meet the needs of use, the cultural heritage of different types of buildings should be preserved in a variety of aspects. Carrascal et al. (2008) established the Cantabria Cultural Heritage Database in Spain based on the standard of CIDOC CRM, and mentioned that the area is rich in various types of cultural heritage, such as artifacts and natural products, and the information has different sources, carriers, and formats. The project integrates this information using the standards established by CIDOC-CRM, showing that CIDOC-CRM covers a wide range of information and can integrate information from different sources. In addition to this case, there are also a number of cultural heritage ontologies extended with CIDOC-CRM as the standard. For example, CRMdig (Doerr and Theodoridou, 2011) focuses on digital data and its sources; Frbroo (Le Boeuf, 2012) expresses the basic semantics of bibliography.

Acierno et al. (2017) designed a ontology model for the management and display of historic preservation materials based on CIDOC-CRM. Its ontology structure considered the concept of life cycle, and added the fields of historic investigation, cultural relics, and personnel. Its ontology model applied it to the building information model, and combined the building component model with the ontology to facilitate the preservation and use of cultural heritage.

Arches is an open source, geospatial concept developed by the World MONUMENTS FUND (WMF) and The Getty Conservation Institute, which is a platform developed for the preservation and management of cultural heritage. Its interpretation data standard also uses CIDOC CRM (Arches, 2019; Myers, et al, 2016). Arches defines six entities of ontology based on CIDOC CRM, as shown in Figure 1, which are: 1 . Historical resources, 2. Historical clusters, 3. Activities, 4. Historical events, 5. Characters and 6. Information resources. Currently, most use cases can be browsed on the official website.

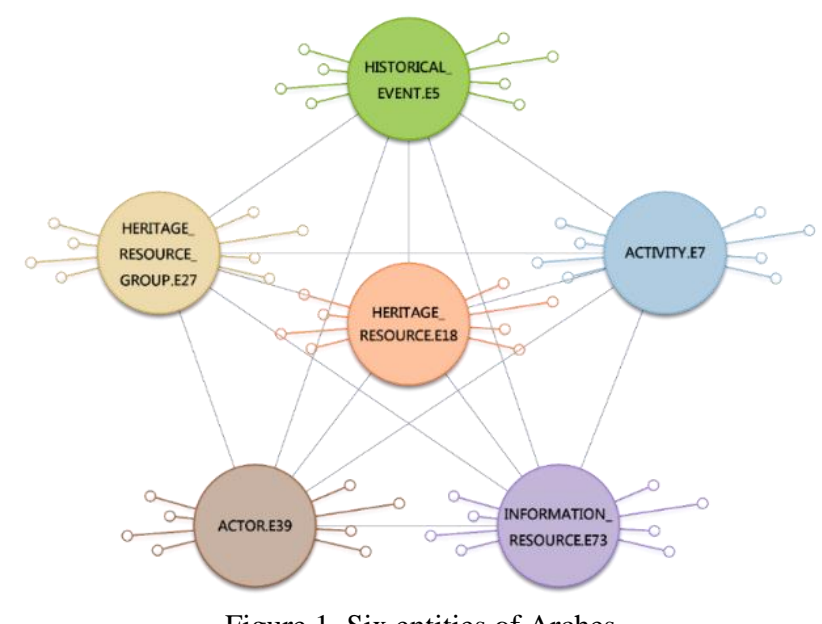

Figure 1. Six entities of Arches

\section{METHODOLOGY}

\subsection{Formulation of metadata}

Cultural heritage as non-renewable resources will likely continue to fade and destroy with time, and the concept of effective preservation of cultural heritage should pay attention to the daily management and maintenance, but also can extend the existence of cultural heritage and the original authenticity. The preservation of cultural heritage should be based on a good management and maintenance system. In order to implement the preservation of cultural heritage in Taiwan, this project will analyze the relevant laws, regulations and methods for the preservation, management and maintenance of cultural heritage in Taiwan. At the same time, take the relevant laws or methods of domestic monuments as the benchmark, so as to establish the requirements for the preservation, management and maintenance of cultural heritage and apply them in the future. Taiwan's "Cultural Heritage Preservation Act" has been in effect for 40 years since it was announced in 1982, and has undergone six amendments, and it is necessary to review and evaluate cultural heritage on a regular basis under the premise of the sustainability of cultural heritage. In accordance with the "Cultural Heritage Preservation Act" issued by the Cultural Heritage Bureau of the Ministry of Culture in 2016, and other subsequent relevant amendments, such as the "Regulations Governing the Review for Designation and Revocation of Monuments", the "Regulations for Maintenance Plan of Monument", and the "Measures for the Examination of the Recognition and Abolition of Folk Customs Registration"; this paper, by means of designated registration, investigation and research, planning and design, inheritance, management and maintenance, promotion, activation as well as three-stage classification and integration of the data, documents, images, sound and others required for the preservation and management of cultural heritage will be carried out to manage the data, digital technology and value-added application of cultural heritage, as shown in Figure 2. 


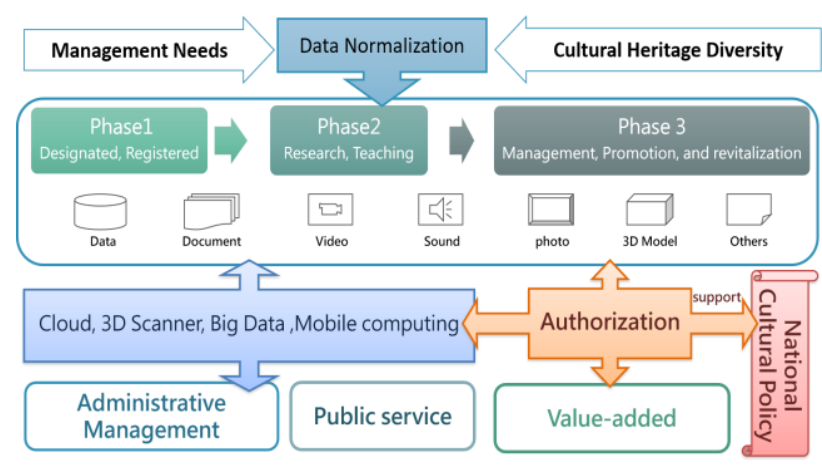

Figure 2. Conceptual diagram of the full life cycle of cultural heritage

This study uses CIDOC CRM ontology to correspond with cultural heritage category attributes, establishes relevant metadata, studies the use of cultural heritage category form fields in the current national cultural heritage network, reviews its shortcomings, holds an expert meeting to discuss and formulate supplementary fields, and expands fields such as architectural structure, style and materials according to expert suggestions, cross compare the form field, extended field and Arches heritage resource class.

\subsection{The establishment of ontology}

Based on the latest version of the international standard CIDOC CRM 6.2, this study constructs the ontology, which includes 89 entity categories and 149 properties. Based on the metadata of the designated registration, management, and maintenance of monuments, folk customs and other categories, the metadata is correspondingly expanded, and the fields are established in accordance with the CIDOC CRM international cultural data exchange standard. Establish an metadata field based on cultural heritage laws and regulations, use it as a basis and conduct a review of its deficiencies, define fields based on the focus group method, and integrate metadata fields, expansion, and other fields with Arches heritage resource classes are crosscompared, and part of the ontology model built after the comparison is shown in Figure 3. For example, the historical site is a main "entity", and different "relationships" are associated with other related entities. Different entities represent different Information. Relevant information is associated with historical settlement as the parent category, so that the relationship between information is presented as an overall relationship between resources.

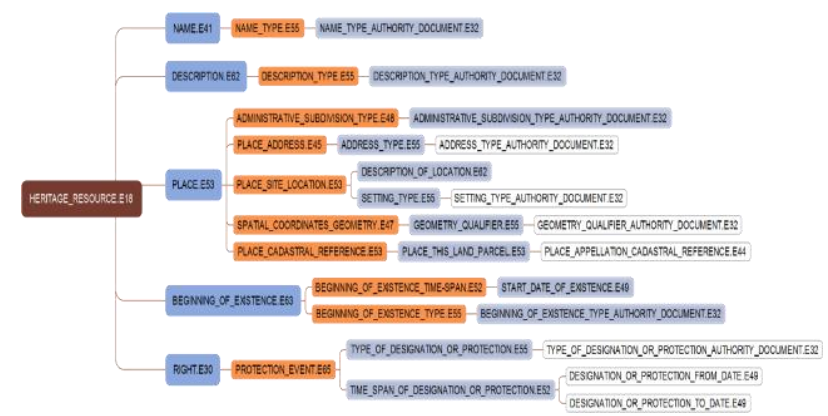

Figure 3. Example architecture diagram of ontology of historical settlement

\begin{tabular}{|c|c|c|}
\hline $\begin{array}{c}\text { CIDOC CRM } \\
\text { category }\end{array}$ & $\begin{array}{l}\text { Literature } \\
\text { database } \\
\text { Field name } \\
\end{array}$ & Content examples \\
\hline $\begin{array}{l}\text { HERITAGE_R } \\
\text { ESOURCE.E18 }\end{array}$ & Case name & $\begin{array}{l}\text { Tsai's Family Ancestral } \\
\text { Temple }\end{array}$ \\
\hline NAME.E41 & $\begin{array}{l}\text { Case name } \\
\text { Case nickname }\end{array}$ & $\begin{array}{l}\text { Tsai's Family Ancestral } \\
\text { Shrines }\end{array}$ \\
\hline $\begin{array}{l}\text { NAME_TYPE_ } \\
\text { AUTHORITY_ } \\
\text { DOCUMENT.E } \\
32\end{array}$ & Category & $\begin{array}{l}\text { Case name } \\
\text { Case nickname }\end{array}$ \\
\hline $\begin{array}{l}\text { HERITAGE_R } \\
\text { ESOURCE_US } \\
\text { E_TYPE.E55 }\end{array}$ & Type & Ancestral shrine \\
\hline $\begin{array}{l}\text { USE_TYPE_A } \\
\text { UTHORITY_D } \\
\text { OCUMENT.E3 } \\
2\end{array}$ & Type list & $\begin{array}{l}\text { Ancestral shrine, temple, } \\
\text { church, mansion, official } \\
\text { residence, shop, castle, } \\
\text { gate, government office, } \\
\text { office, office building, } \\
\text { bank, assembly hall, } \\
\text { market,............. }\end{array}$ \\
\hline & & \\
\hline
\end{tabular}

Table 1. Example of mapping

In this study, the software of Protégé is used to establish and display the ontology model. The ontology model is established by the software for understanding and exchanging; the main function is to establish the category and relevance of the ontology, so that the ontology model is built, and the structure of the display model can enable the non-professional field personnel to quickly understand the concept that the field and the model builder want to convey.

The types of ontology models designed in this study are relatively complex. In order to facilitate the understanding of the relevance of entities, this paper presents a graphical representation of Figure 4 as the database ontology model built in this study. As mentioned in the previous paragraph, from the middle of the Heritage Resource_E18 entity expand outward and are associated with entities such as name and important date to form a network ontology model. The characteristics of the model are easy to understand. Users can understand the design concept and operation mode of the model only by examining the category and relationship definition in the ontology. Compared with the forms and key values of the previous database, the model is more clear and clear, and the data interchangeability is improved as a whole. 


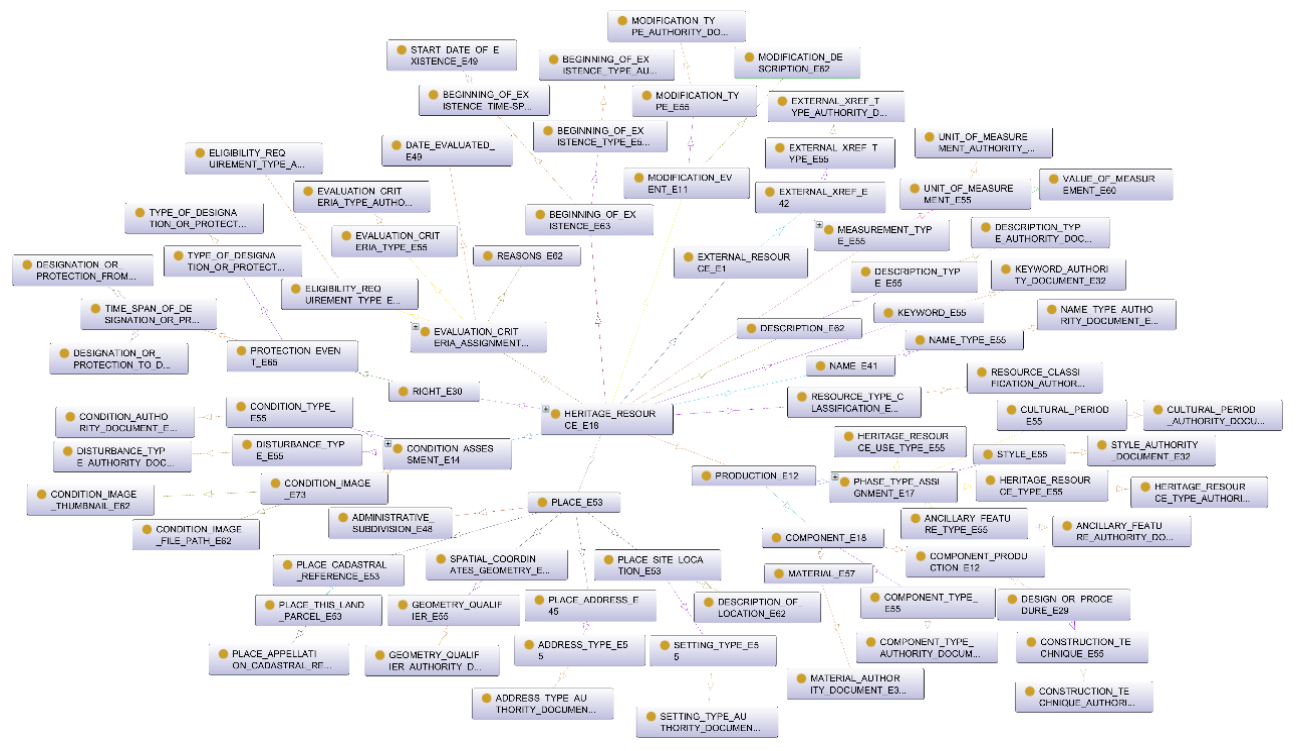

Figure 4. Ontology model of historic site

\section{SYSTEM CONSTRUCTION}

\subsection{Qiong Lin Settlement in Kinmen}

This study takes the Kinmen's Qiong Lin Settlement as a case study (Figure 5). Qiong Lin Settlement has rich tangible cultural heritage and intangible cultural heritage, intangible cultural heritage Tsai's Family Ancestral Temple of Qiong Lin Village's family temple festival is held in the national monument Tsai's Family Ancestral Temple, indicating the inseparable link of tangible and intangible cultural heritage. Qiong Lin Settlement also has many cultural heritage, including national historic monuments, county historic monuments, historical buildings, settlement buildings, folklore and so on.

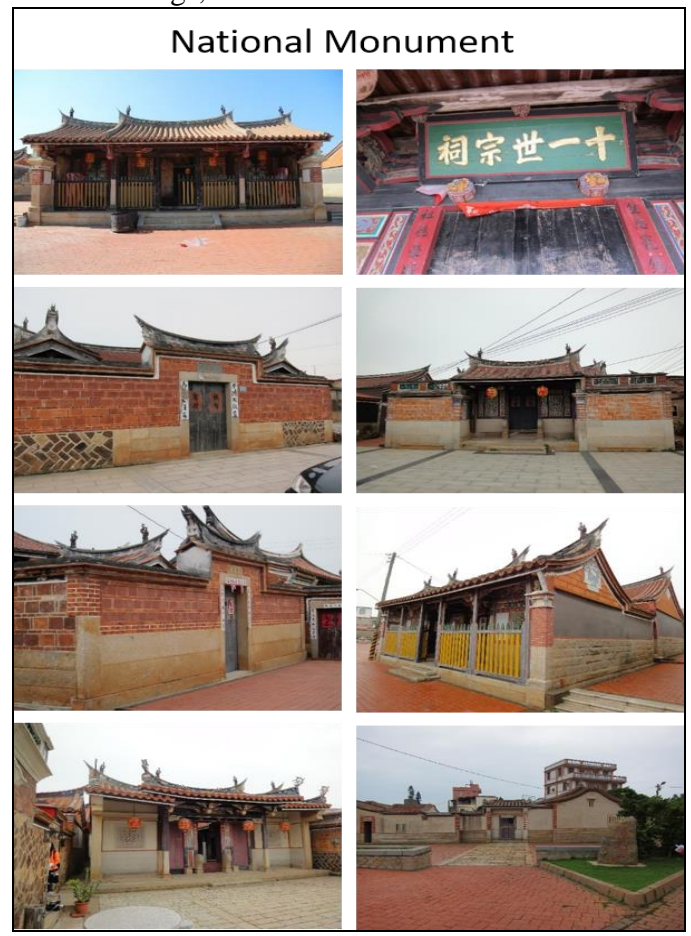

Figure 5. Qiong Lin Settlement

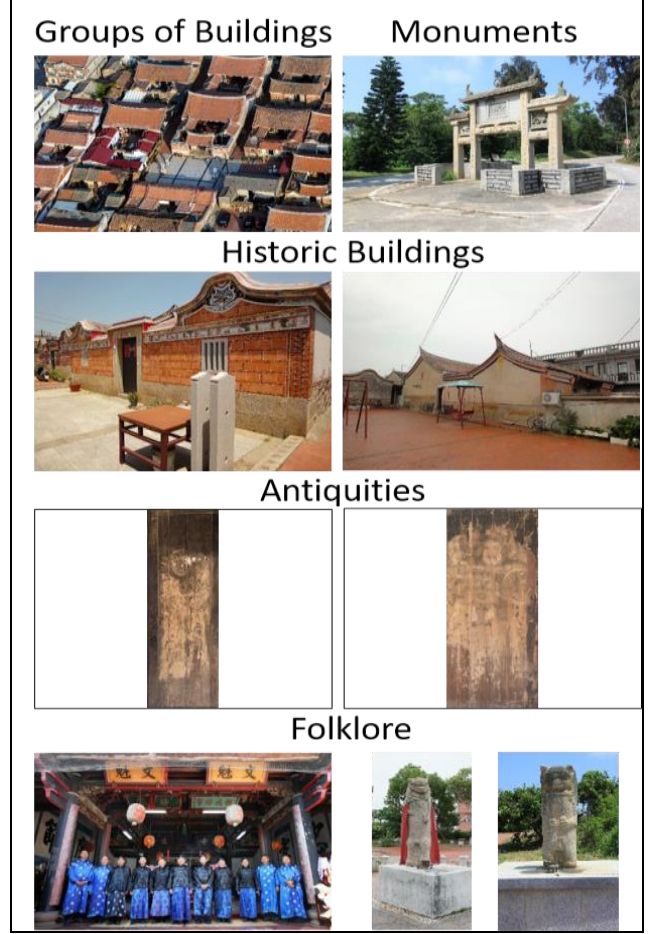

Figure 5. Qiong Lin Settlement

\subsection{Arches system platform}

The home page of Arches system platform is shown as Figure 4, and the user can display the main tabs, customized pictures and quick search functions on the home page; click the search navigation tab to enter the query page; the login tab is the entrance for the administrator to enter the background, various types of ontology and data can be built; at the bottom of the home page (Figure 6), statistics (Figure 7) and information or important links (Figure 8) related to cultural heritage can be displayed. 


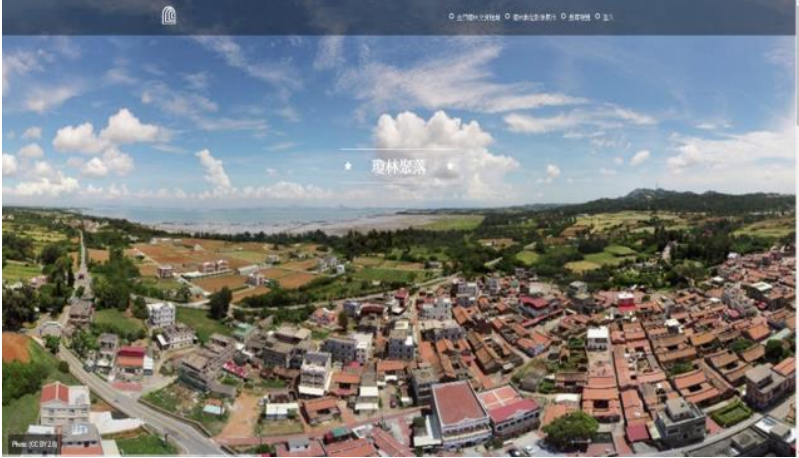

Figure 6.System platform on home page

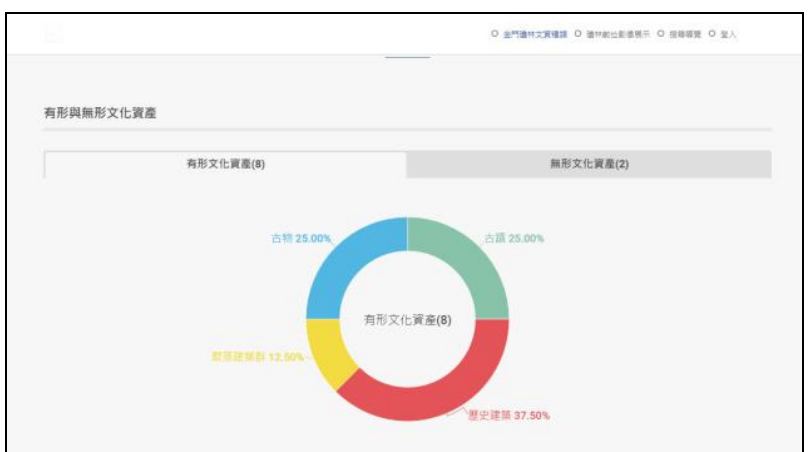

Figure 7. Statistics function of system

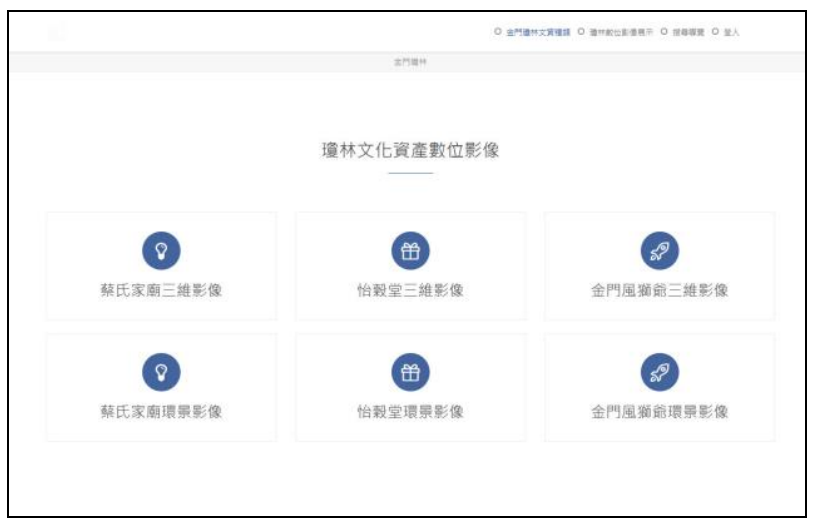

Figure 8. Connection function of system

This study uses the Arches platform based on CIDOC CRM, and takes the cultural heritage of Qiong Lin Settlement in Kinmen as an example, including 1.Heritage Resource; 2.Heritage Resource Group; 3.Activity; 4.Historical Event; 5.Actor; 6. Information Resource etc. After integrating the ontology model, we use the above entities to construct historical settlement, historical buildings, settlement complexes, competent authorities, owners, management, and maintenance, major issues, management and maintenance plans, education and training, community participation, etc. In the case of Qiong Lin Settlement in the above entities (Figure9), after completing the creation of the category, fill in the case-related information (Figure 10), and then specify the association between the case and the case through the complete fill-in, and then directly view the case related to the category when inquiring in the system.

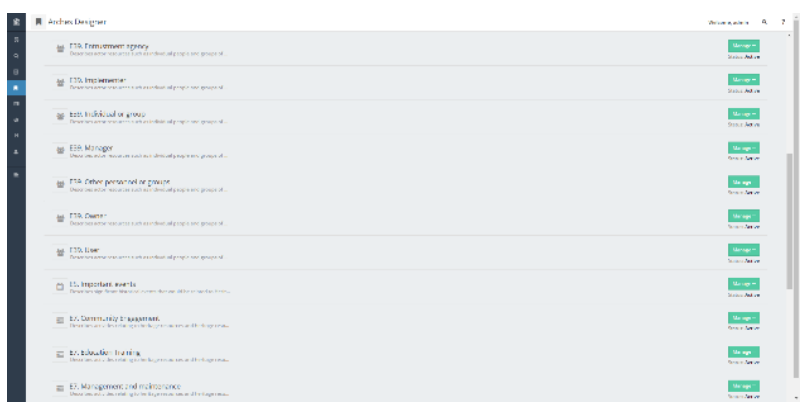

Figure 9. Display chart of home page connection function
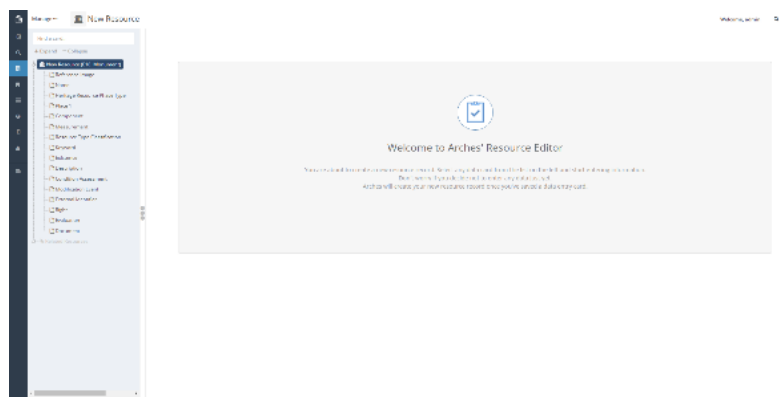

Figure 10. Display chart of home page connection function

After the establishment of the relevant information about the Tsai's Family Ancestral Shrines, a national historic site in the Qiong Lin Settlement, users can browse and search for resources on the data search page. The main search page is initially set to map search (Figure 11), or after searching for the historic site of Yi Gu Tang by category, Click related resources to display a tree-like resource association diagram (Figure 12) of related resources. Other nodes in the tree diagram represent resources related to $\mathrm{Yi} \mathrm{Gu}$ Tang. The resources may be fields, people, things, objects, etc.

The function of the Arches platform allows users who are not familiar with the cultural heritage to quickly understand the resources related to it, and users who are already familiar with it can quickly search for the required data. After the Arches platform is imported according to the ontology model, browse the whole concept of the web browsing model, and can also save a lot of manpower and output into multiple web languages for different purposes.

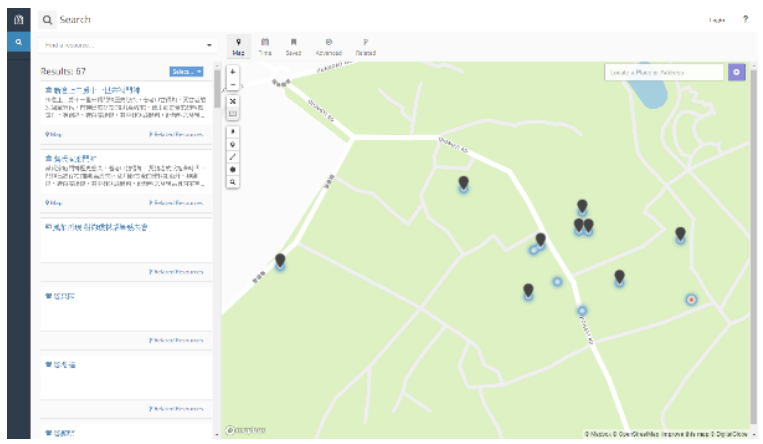

Figure 11. Map resource search function map 


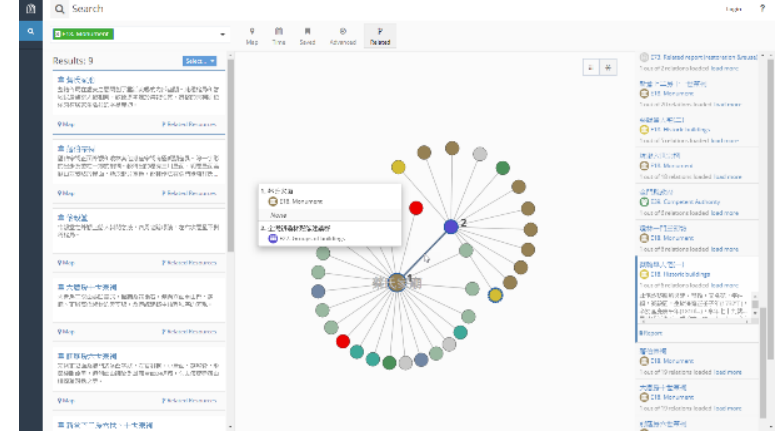

Figure 12. Association diagram of related resources of Yi Gu Tang

\section{CONCLUSIONS AND FUTURE WORK}

This study takes the cultural heritage category in the Qiong Lin Settlement of Kinmen as the main research object, and establishes the metadata for the preservation of cultural heritage based on the ontology, including related objects, space and other related cultural heritage. In the future, we should strengthen the accuracy of filling in the forms related to cultural heritage, which will help to improve the integrity and relevance of the data content.

Based on the implementation results of the ontology of this study, the entire life cycle of the ontology of Taiwan's cultural heritage can be completely built, such as planning, restoration, inheritance, promotion, and activation. Connecting tangible and intangible cultural resources in accordance with international data standards, vertical and horizontal integration will help to meet the needs of data retrieval and administrative management. This project has completed the ontology framework of cultural heritage integration at the present stage. It is suggested that it should continue to expand to other types of cultural heritage, and introduce a broader framework of international cultural heritage types (such as world memory, underwater cultural heritage, world heritage, intangible heritage, etc.). The trend of international cultural heritage preservation should be promoted, and this study should establish a ontology framework based on international standards and a presentation platform using semantic technology, which can be used as a requirement for international data exchange and discussion of subsequent application specifications in the future.

\section{ACKNOWLEDGEMENTS}

Thanks for the supports from Ministry of Science and Technology, the Interdisciplinary Research Project, MOST109-2625-M-163 -001, and the Bureau of Cultural Heritage, Ministry of Culture, the College of Cultural Heritage Project, $109-\operatorname{Re}-12$ to this study.

\section{REFERENCES}

Aciernoa, M., Cursib, S., Simeoneb, D and Fiorania, D., 2017. Architectural heritage knowledge modelling: An ontologybased framework for conservation process. Journal of cultural heritage, 24, pp. 124-133.

Arches, 2019. What is Arches? Retrieved June 25, 2021, from https://www.archesproject.org/what-is-arches/.

CIDOC-CRM. Retrieved June 25, 2021, from http://www.cidoc-crm.org/.
Doerr, M. 2009. Ontologies for cultural heritage. Handbook on ontologies Springer, Berlin, Heidelberg. pp. 463-486.

Doerr, M., and Theodoridou, M., 2011. CRMdig: A generic digital provenance model for scientific observation. TaPP'11 3rd USENIX Workshop on the Theory and Practice of Provenance, Heraklion, Greece, June 20-21, 2011.

Hernández-Carrascal, F. 2008. Ontología del patrimonio de Cantabria. Profesional de la Información, 17(1), 92-98.

Le Boeuf, P. 2012. An example of library and museum cooperation: the FRBROO conceptual model. In CIDOC Annual Conference, Enriching Cultural Heritage, Helsinki, Vol. 10, No. 14.06.

Ministry of Culture, 2016. Cultural Heritage Preservation Act, Chapter 1, Article 3.

Moraitou, E., Aliprantis, J., Christodoulou, Y., Teneketzis, A., \& Caridakis, G. 2019. Semantic Bridging of Cultural Heritage Disciplines and Tasks. Heritage, 2(1), 611-630.

Myers, D., Quintero, M. S., Dalgity, A., \& Avramides, I. 2016. The Arches heritage inventory and management system: a platform for the heritage field. Journal of Cultural Heritage Management and Sustainable Development.

Ronzino, P., Niccolucci, F., \& D'Andrea, A. 2013. Built Heritage metadata schemas and the integration of architectural datasets using CIDOC-CRM. In Online proceedings of the International Conference Built Heritage. 